\title{
Algebra dissociates from arithmetic in the brain semantic network
}

\section{Dazhi Cheng}

Beijing Normal University

Mengyi Li

Beijing Normal University

Naiyi Wang

Beijing Normal University

Liangyuan Ouyang

Tianjin Normal University

Xinlin Zhou ( $\square$ zhou_xinlin@bnu.edu.cn )

Beijing Normal University https://orcid.org/0000-0002-5570-2240

\section{Research}

Keywords: Algebra, Arithmetic, Semantic network, Phonological network, Visuospatial network

Posted Date: August 19th, 2021

DOl: https://doi.org/10.21203/rs.3.rs-806057/v1

License: (c) (1) This work is licensed under a Creative Commons Attribution 4.0 International License.

Read Full License

Version of Record: A version of this preprint was published at Behavioral and Brain Functions on January 7th, 2022. See the published version at https://doi.org/10.1186/s12993-022-00186-4. 


\section{Abstract \\ Background}

Mathematical expressions mainly include arithmetic (such as $8-(1+3)$ ) and algebraic expressions (such as $a-(b+c)$ ). Previous studies shown that both algebraic processing and arithmetic involved the bilateral parietal brain regions. Although behavioral and neuropsychological studies have revealed the dissociation between algebra and arithmetic, how algebraic processing is dissociated from arithmetic in brain networks is still unclear.

\section{Methods}

Using functional magnetic resonance imaging (fMRI), this study scanned 30 undergraduates and directly compared the brain activation during algebra and arithmetic. Brain activations, single-trial (item-wise) interindividual correlation and mean-trial interindividual correlation related to algebra processing were compared with those related to arithmetic.

\section{Results}

Brain activation analyses showed that algebra elicited greater activation in the angular gyrus and arithmetic elicited greater activation in the bilateral supplementary motor area, left insula, and left inferior parietal lobule. Interindividual single-trial brain-behavior correlation revealed significant brain-behavior correlations in the semantic network, including the middle temporal gyri, inferior frontal gyri, dorsomedial prefrontal cortices, and left angular gyrus, for algebra. For arithmetic, the significant brain-behavior correlations were located in the phonological network, including the precentral gyrus and supplementary motor area, and in the visuospatial network, including the bilateral superior parietal lobules.

\section{Conclusion}

These findings suggest that algebra relies on the semantic network and arithmetic relies on the phonological and visuospatial networks.

\section{Background}

Mathematical expressions are frequently conceived and represented in arithmetic and algebraic expressions. Arithmetic is concretely represented as the combination of digits and operators (such as 8 $(1+3))$. Contrastingly, algebra includes abstract operations with letters and operators (such as a - $(b+$ c)) and has more abstract representation than arithmetic in form [1]. Although behavioral and neuropsychological studies have reported that algebra can be dissociated from arithmetic [2-8], the neural 
mechanisms underlying this dissociation remain unclear. The current study used fMRI to examine how algebraic processing dissociates from arithmetic processing in brain networks.

\section{Arithmetic in the brain}

In the arithmetic expression " $4 \times 5-9$," the sub-expression " $4 \times 5$ " is recursively combined with " 9 " by subtraction. It has been proposed that the arithmetic operations both involved numerical quantity calculation and order-rule of arithmetic operations [9]. Neuroimaging and neuropsychological studies have revealed that arithmetic processing is subserved by the visuospatial network, which typically includes the intraparietal sulcus (IPS) and inferior and superior parietal lobules [10-15]. Several studies have shown that both arithmetic and number processing are mediated by common regions in the parietal lobules [14, 16-18]. Approximately $73 \%$ of the behavioral variance of visuospatial magnitude processing has been attributed to the change in cortical thickness in the right superior parietal cortex, and approximately $55 \%$ of the behavioral variance of children's arithmetic abilities has been attributed to the change in cortical folding in the right IPS [19]. Patients with injuries in the parietal cortex typically experience arithmetic impairment $[20,21]$ and impaired visuospatial performance [22].

During arithmetic computation, the phonological system also supports the coding of visual or auditory numbers, as well as the temporary storage of intermediate results $[23,24]$. Therefore, arithmetic processing also relies on the phonological network, which typically includes the bilateral supplementary motor areas and the precentral gyrus [15, 16, 25-28]. For example, Zhou et al. [28] reported that both addition and multiplication are supported by a broad neural system involving the supplementary motor areas, precentral gyrus, IPS, and middle frontal gyrus. One recent study showed that exact arithmetic involves more phonological or verbal processing localized in the left Rolandic operculum, precentral gyrus, and supplementary motor area than approximate arithmetic [26]. Neuropsychological investigations have also shown that patients with infarction in the left frontal lobe (including the precentral gyrus) have difficulty retrieving arithmetic facts $[29,30]$.

\section{Algebra in the brain}

Algebraic expressions, such as $a^{2}+b^{2}=c^{2}$, have a nested hierarchical structure of arithmetic procedural knowledge $[9,31]$. Previous imaging studies have revealed that regions in the parietal lobes respond to both algebra and arithmetic. The brain organization of algebra is dissociated from language processing [32-34], which is similar to the dissociation between basic numerical processing and language processing $[12,13,35,36]$. Specifically, algebraic processing typically recruits the bilateral parietal brain regions around the IPS to represent numerical magnitude, whereas processing the syntax of language elicits activation in the classic language areas including the left inferior frontal gyrus and the posterior and anterior middle temporal gyrus [34]. 
However, behavioral $[2,6,7]$ and neuropsychological $[3-5,8]$ studies further revealed the dissociation of algebraic abilities and arithmetic processing. For example, preschool children can complete three-term arithmetic inversion problems (such as $3+2-2$ ) but do not understand the inversion principle of algebra $(a+b-b)$ (Rasmussen et al., 2003). One neuropsychological study reported that a patient with Fahr's disease with both basal ganglia and frontal lobe brain damage was able to solve most simple subtraction and addition problems but could not understand algebraic concepts (such as $a+b=b+$ a) [8]. These findings imply that there may be dissociated brain neural bases between algebra and arithmetic.

Algebraic expressions also involves language symbolic and represents abstract mathematical cognitions, which requires the application of conceptual and semantic knowledge [12, 32, 37, 38]. For example, Geary et al. [38] found that algebraic cognition was related to semantic memory systems of addition facts in ninth graders. Neuroimaging studies further implied that the brain network's response to algebra is also associated with language semantic processing. Recent studies have shown that compared to simple numerical processing and arithmetical computation, mathematical semantic knowledge (e.g., arithmetic principles and algebraic terms) elicited greater activation in the typically semantic network such as left angular gyrus, left middle temporal gyrus, and left inferior frontal gyrus [12, 39]. These regions typically overlap with the semantic network, including the posterior inferior parietal lobule (angular gyrus), middle temporal gyrus, inferior frontal gyrus, dorsomedial prefrontal cortex, fusiform and parahippocampal gyri, ventromedial prefrontal cortex, and posterior cingulate gyrus [40].

\section{The present study}

Although behavioral $[2,6,7]$ and neuropsychological $[3-5,8]$ studies have reported that algebra is dissociated from arithmetic, how algebraic processing dissociated from arithmetic processing in brain networks is unclear. This study uses fMRI to identify the specific neural bases for algebraic processing and arithmetic processing. The arithmetic and algebra tasks used in this study were matched in surface form and difficulty. In the present study, algebra used the operational principles for addition, subtraction, multiplication, and division (such as a- $(b+c)$ ). To match the algebraic tasks, we used tasks requiring arithmetic procedures (such as $8-(1+3)$ ) rather than tasks requiring the simple retrieval of arithmetic facts.

Prior studies have indicated that arithmetic involves numerical quantity operations and phonological processing [10-15]. Contrastingly, the application of semantic knowledge is involved in algebraic processing [12]. Therefore, we hypothesized that algebra relies more on the semantic network and arithmetic involves the phonological and visuospatial networks based on the aforementioned reports. To test this hypothesis, we compared the fMRI data regarding brain activation for algebra with that for arithmetic, and we conducted a single-trial (item-wise) interindividual correlation analysis and a traditional mean-trial interindividual correlation analysis to examine the brain-behavior correlations [26, 41]. 


\section{Methods}

\section{Participants}

Thirty right-handed undergraduate students ( 15 males and 15 females; mean age $=21.92$ years, range: 18-25 years) were recruited to participate in this study from Beijing Normal University, China. They majored in a wide range of disciplines except for mathematics. These participants reported having no history of neurological disorders or head injuries. The experiment was fully explained to the participants before informed consent was obtained. This study was approved by the Institutional Review Board of the Institute of Cognitive Neuroscience and Learning at Beijing Normal University.

\section{Materials}

The algebraic task included the operational principles for addition, subtraction, multiplication, and division acquired by students in primary school. The arithmetic task included the numerical calculations for addition, subtraction, multiplication, and division. Each problem included two expressions, and the average length of the problems was controlled (Fig. 1). All expressions were presented in white against a black background (with a red, green, and blue value of $0,0,0$ ). The participants were given 16 algebra and 16 arithmetic problems to complete.

The stimulus presentation and behavioral data recordings were programmed using E-prime software (Version 1.1, Psychology Software Tools, Inc., www.pstnet.com) on a Pentium 4 laptop. Stimuli were projected onto a translucent screen placed at the back of the magnet bore. Participants viewed the screen through a mirror mounted on the head coil at a distance of $30 \mathrm{~cm}$ from the eyes.

\section{Procedure}

Prior to scanning, the participants underwent a training session with the same type of materials as a formal experiment to ensure that they understood the instructions of this experiment. After that, participants were required to complete the experimental tasks in the scanner.

The scanning session was organized into two runs, each lasting $192 \mathrm{~s}$. Each run consisted of four experimental blocks (two experimental blocks for each task) and four baseline blocks (Fig. 1). The balanced Latin square design [42] was used to counterbalance the order effect of the two experimental tasks. Each block with four trials lasted for $24 \mathrm{~s}$, and the presentation order of trials was random. There was a 1-min rest period after each run.

For each trial in the experimental blocks, two expressions were presented synchronously on the screen, one at the top and one at the bottom. Participants were asked to judge whether the two expressions were equal. For each trial in the baseline blocks, two arrows were presented synchronously on the screen, one at the top and one at the bottom. Participants were asked to judge whether the two arrows pointed in the 
same direction. Each trial lasted $6 \mathrm{~s}$, and a fixation cross was displayed to fill the remaining time if the participant did not use all $6 \mathrm{~s}$. Half of the participants responded to the trials by pressing a key on a response box on their left with their left index finger when the two mathematical expressions or arrows were equal. The remaining participants responded to the trials by pressing a key on a response box on their right with their right index finger. Both accuracy and speed were emphasized.

\section{fMRI data acquisition}

Images were obtained using a Siemens (Munich, Germany) 3T Trio MRI scanner using a standard eightchannel head coil. After automatic shimming of the magnetic field, three-dimensional high-resolution T1 anatomical images were acquired for co-registration with the functional images. Next, functional volumes were acquired using a multiple slice $\mathrm{T} 2^{*}$-weighted echo planar imaging (EPI) sequence with the following parameters: in-plane resolution $=3.125 \times 3.125 \mathrm{~mm}^{2}$; repetition time $=2000 \mathrm{~ms}$; echo time $=30 \mathrm{~ms}$; flip angle $=90^{\circ}$; field of view $=240 \times 240 \mathrm{~mm}^{2} ;$ matrix dimensions $=64 \times 64$; field of view $=200 \mathrm{~mm}$; and slice thickness $=4 \mathrm{~mm}$. The entire brain was imaged in 32 slices.

\section{Statistical analysis of the fMRI data}

Individual MRI datasets were analyzed using SPM12 software (Wellcome Department of Imaging Neurosciences, University College London, UK; http://www.fil.ion.ucl.ac.uk/spm). All volumes were realigned to the first volume and spatially normalized to a common value to correct for whole brain differences over time. Images were then smoothed using an isotropic Gaussian kernel of $4 \mathrm{~mm}$ and highpass filtered at a cut-off of $128 \mathrm{~s}$.

Brain activation analysis. After preprocessing, parameter-estimated images for individual participants across the whole brain were calculated. Group analyses with random effects were conducted using the one-way analysis of variance (ANOVA) on the brain activation maps of all participants with material type as the independent variable. Then, the brain activation for each type of material relative to fixation was calculated. Brain activations for the two types of materials were compared. We used the thresholds from the lenient $p<.05$ to $p<.005$, to the stringent $p<.001$, uncorrected, with a minimum cluster size of 20 voxels.

Brain-behavior correlation analysis. A single-trial (item-wise) interindividual correlation analysis that has been reported in previous fMRI and event-related potential (ERP) studies was used to examine the brainbehavior correlations ( $\mathrm{Li}$ et al., 2020; Zhou et al., 2018). First, the correlation between the brain activation maps and the reaction times (RTs) was determined for each trial. Then, a one-sample $t$-test on the correlation coefficients obtained for all trials of one type of processing against zero was performed. The analysis was performed separately for algebra and arithmetic tasks. The single-trial correlation has been reported as more effective than the traditional mean-trial correlation as it can filter out much of the noise that exists after the first step [41]. 
The traditional mean-trial interindividual correlation was also used, and the results were compared with those of the single-trial correlation. The mean brain activation map and the mean RT for each type of processing for each participant were determined. Then, a correlation analysis between the mean brain activation map and the RT for all participants was performed. We used the thresholds for two brainbehavior correlation analyses from the lenient $p<.05$ to $p<.005$, to the stringent $p<.001$, uncorrected, with a minimum cluster size of 20 voxels.

Further, we defined seven functional regions of interest (ROIs) based on results from previous studies for the semantic, phonological, and visuospatial networks. Based on the meta-analysis of 120 functional neuroimaging studies of semantic processing (Binder et al., 2009), we defined four ROls of the semantic network in the left hemisphere that included the middle temporal gyrus (Montreal Neurological Institute (MNI) coordinates $[-45,-21,-15])$, inferior frontal gyrus (MNI coordinates $[-51,24,0])$, dorsomedial prefrontal cortex (MNI coordinates [-6, -48,30]), and angular gyrus (MNI coordinates [-57, -45, 30]). Based on the fMRI studies of phonological processing for arithmetic (Zhou et al., 2007), we defined two ROIs of the phonological network in the left hemisphere that included the precentral gyrus (MNI coordinates $[-45,4,30])$ and supplementary motor area (MNI coordinates $[-3,3,58])$. Based on the fMRI studies of visuospatial processing for arithmetic [43], we defined one ROI of the visuospatial network in the left superior parietal lobule (MNI coordinates $[-32,-68,56])$. Each ROI was a sphere with a radius of 9 $\mathrm{mm}$. These ROls were used to compare the levels of brain-behavior correlations between algebra and arithmetic.

\section{Results}

\section{Behavioral results}

The mean RT was $2653 \mathrm{~ms}$ for algebraic task and $2855 \mathrm{~ms}$ for arithmetic task. The mean accuracy was $87.6 \%$ and $86.8 \%$, respectively. RTs and accuracy were analyzed with a repeated measure ANOVA. The main effect of stimulus type was not significant for $\mathrm{RTs}\left(F(1,29)=2.55, p=.116, \eta^{2}=.042\right)$ or accuracy $\left(F(1,29)=1.10, p=.754, \eta^{2}=.002\right)$.

\section{Brain activations}

The brain activations for algebra and arithmetic relative to baseline are displayed in Fig. 2. Algebra and arithmetic elicited significant activation in a broad array of brain regions, including the bilateral parietal, frontal, and occipital gyri. The differences in brain activation between algebra and arithmetic are shown in Fig. 2 and Table 1. Algebra relative to arithmetic had greater activation in the bilateral angular gyrus. In contrast, arithmetic elicited greater activation in the bilateral supplementary motor areas, left insula, and left inferior parietal lobule than algebra.

\section{Brain-behavior correlations}


The single-trial brain-behavior correlations for algebra and arithmetic are displayed in Fig. 3 and Table 2. Significant positive brain-behavior correlations $(p<.001$, uncorrected) were observed in the bilateral middle temporal gyri, bilateral inferior frontal gyri, bilateral dorsomedial prefrontal cortices, and left angular gyrus for algebra. Significant negative brain-behavior correlations $(p<.001$, uncorrected) were observed in the bilateral superior parietal lobules for algebra. For arithmetic, significant positive brainbehavior correlations ( $p<.001$, uncorrected) were observed in the right inferior temporal gyrus and negative brain-behavior correlations ( $p<.001$, uncorrected) were observed in the bilateral superior parietal lobules, bilateral middle occipital gyri, bilateral precentral gyri, left supplementary motor area, left insula, and bilateral cerebella. Fig. 3 shows the number of voxels of the single-trial brain-behavior correlations for algebra and arithmetic in the ROls used in this study. Algebra elicited a larger number of voxels in four ROls of the semantic network, including the middle temporal gyrus, inferior frontal gyrus, dorsomedial prefrontal cortex, and angular gyrus. Arithmetic elicited a greater brain-behavior correlation and a larger number of voxels in three ROls of the phonological and visuospatial networks, including the precentral gyrus, supplementary motor area, and superior parietal lobule. The differences between algebra and arithmetic can found based on the single-trial brain-behavior correlation approach under the threshold $p<$ .001 , uncorrected.

The traditional mean-trial brain-behavior correlations for algebra and arithmetic are displayed in Fig. 4 . Significant positive brain-behavior correlations ( $p<.05$, uncorrected) were observed in the bilateral middle temporal gyri and bilateral dorsomedial prefrontal cortices, and negative brain-behavior correlations ( $p<$ .05 , uncorrected) were observed in the bilateral superior parietal lobules for algebra. Significant positive brain-behavior correlations ( $p<.05$, uncorrected) were observed in the bilateral inferior temporal gyri, and negative brain-behavior correlations ( $p<.05$, uncorrected) were observed in the bilateral superior parietal lobules, bilateral middle occipital gyri, bilateral precentral gyri, and bilateral cerebella for arithmetic. Fig. 4 shows the number of voxels of the mean-trial brain-behavior correlations for algebra and arithmetic for each ROI, which identifies weak differences between algebra and arithmetic under the threshold $p<.05$, uncorrected.

\section{Discussion}

The present study aimed to clarify the neural dissociation between algebra and arithmetic. Betweengroup brain activation analyses revealed that algebra resulted in greater activation in the bilateral angular gyrus, and arithmetic elicited greater activations in the bilateral supplementary motor area, left insula, and left inferior parietal lobule. Interindividual single-trial brain-behavior correlation analyses showed significantly positive brain-behavior correlations in the semantic network, including the bilateral middle temporal gyri, bilateral inferior frontal gyri, bilateral dorsomedial prefrontal cortices, and left angular gyrus for algebra. For arithmetic, the significantly negative brain-behavior correlations were observed in the phonological network, including the bilateral precentral gyrus and left supplementary motor area, and in the visuospatial network, including the bilateral superior parietal lobules. These findings suggest that algebra relies more on the semantic network and arithmetic relies more on the phonological and visuospatial networks. 


\section{Semantic network in algebra}

The current study found that algebra relies more on the semantic network, including the bilateral angular gyrus, bilateral middle temporal gyri, bilateral inferior frontal gyri, bilateral dorsomedial prefrontal cortices, and left angular gyrus, than arithmetic. These regions overlap with the semantic network according to the meta-analysis of functional neuroimaging studies by Binder et al. that was based on word processing [40]. In algebra, formula derivations of the relationship between the letter and the operator (such as the commutative or associative laws) require knowledge of mathematical principles. Thus, algebra is similar to mathematical principles. Previous studies have shown that the mathematical principle processing based on conceptual knowledge is subserved by a semantic network $[12,39,44,45]$. Liu et al. [45] reported that mathematical principles (e.g., exchanging the position of two operands in addition does not change their sum) elicited greater activation of the semantic network in the left middle temporal gyrus, left inferior frontal gyrus, and left angular gyrus than arithmetic computation (e.g., when the number 8 is first divided by 4 and then multiplied by 3 , the final result is 6 ). The important role of the semantic network in mathematical principles suggests an important role of conceptual knowledge in algebra.

Furthermore, algebra requires abstract conceptual knowledge, and arithmetic involves concrete conceptual knowledge. Recent studies have suggested that concepts are organized in the brain according to semantic categories [46] such as animate versus inanimate [47] or concrete versus abstract [48] and that the classical semantic network is important for abstract conceptual knowledge [49-51]. A metaanalysis of studies regarding abstract concepts revealed that abstract concepts tend to produce stronger activation in the semantic network than concrete concepts [51]. By contrast, previous studies emphasized that abstract algebra concepts induced the non-linguistic cortical network dissociated from the classical semantic network [32,33]. The subjective representation of the concreteness of algebra by expert mathematicians relied more on mental imagery, which differs from nonmathematical concepts in these studies [32, 33]. However, for laymen, algebra relies on the classical semantic network, suggesting a close association between algebra and language processing.

\section{Phonological and visuospatial networks in arithmetic}

This study found that arithmetic relied more on the phonological network, including the bilateral precentral gyri and supplementary motor areas, than algebra. Matching tasks of arithmetic associated with the retrieval of arithmetic facts are thought to be stored as verbal codes [11] and are related to cognitive and neural processes involved in phonological processing. These results are in line with models of arithmetic operations that outline a phonological processing network [15, 26, 52].

Our results also indicate that arithmetic results in greater activations in the visuospatial networks including the bilateral superior parietal lobules, which is consistent with the previously reported role of the visuospatial network in arithmetic [10-15]. These regions are responsible for visuospatial information 
from the symbolic form of the arithmetic expression and reflect the visuospatial processing of concrete symbolic and quantitative representations in arithmetic. Numbers can elicit a visuospatial response, as the quantity of a number can be represented on a mental number line from left to right. This processing is evidenced by the spatial numerical association of response codes effect, which describes that people respond faster to small numbers with the left hand and large numbers with the right hand [53, 54]. Arithmetic can be completed using the visuospatial processing of a dynamic mental number line, as addition leads to a right shift and subtraction leads to a left shift in visuospatial processing [55-57].

\section{Significance of the single-trial brain-behavior correlation}

Neuroimaging researchers often use interindividual brain-behavior correlations to explore the associations between the brain and human behaviors [58-60]. The correlation analysis investigates how interindividual differences in brain functions relate to interindividual behavioral performances and can help provide an accurate and representative neural mechanism of cognitive processing [61].

In this study, the traditional mean-trial brain-behavior correlations approach yielded correlations with $p<$ .05 , which is not considered significant in fMRI studies. The mean-trial correlation approach may not efficiently remove enough of the noise and does not result in a high correlation coefficient [62]. To improve the disadvantages of the traditional mean-trial correlation approach, we used the single-trial brain-behavior correlation approach previously reported in fMRI and ERP studies [41, 59]. The single-trial brain-behavior correlation approach avoids the influence of noise on the statistical analyses. This correlation approach first removes much of the noise (or residual activity) when determining the correlation for each trial and then conducts a $t$-test for the weak yet possibly stable correlation coefficients with the majority of the noise filtered. The results of this study showed significant brainbehavior correlations in the semantic network for algebra $(p<.001)$ and significant brain-behavior correlations in the phonological and visuospatial networks for arithmetic $(p<.001)$.

\section{Conclusion}

Algebra is the abstract form of arithmetic and activates the semantic network, including the bilateral middle temporal gyri, bilateral inferior frontal gyri, bilateral dorsomedial prefrontal cortices, and left angular gyrus. In contrast, arithmetic activates the phonological network, including the bilateral precentral gyri and supplementary motor areas, and the visuospatial network, including the bilateral superior parietal lobules. These results suggest that the semantic network in the brain supports algebra. It deepened our understanding of the relation between algebraic processing and arithmetic processing. Whereas previous studies suggested that algebraic processing activated the non-semantic cortical network in expert mathematicians [32,33], our results highlighted the important role of the classical semantic network in algebraic processing. In school education in general, algebra learning might take advantage of semantic knowledge. 


\section{Abbreviations}

IPS: intraparietal sulcus; fMRI: functional magnetic resonance imaging; EPI: echo planar imaging; ANOVA analysis of variance; ERP: event-related potential; RTs: reaction times; MNI: Montreal Neurological Institute

\section{Declarations}

\section{Acknowledgements}

Not applicable.

\section{Author Contributions}

D.Z., M.Y. and X.L. conceived and designed the study. D.Z., M.Y. and L.Y. performed the experiment and analyzed the data. D.Z. and M.Y. interpreted the data and drafted the manuscript. N.Y. and X.L. critically revised the manuscript for important intellectual content. All authors have read and approved the manuscript.

\section{Funding}

This research was supported by grants from the Natural Science Foundation of Beijing (grant number: 5212004), National Natural Science Foundation of China (grant number: 31700977, 31671151), the 111 Project (grant number: BP0719032), and the Advanced Innovation Center for Future Education (grant number: 27900-110631111).

\section{Availability of data and materials}

The datasets are available from the corresponding author on formal and logical request.

\section{Ethics approval and consent to participate}

This study was approved by the Institutional Review Board of the Institute of Cognitive Neuroscience and Learning at Beijing Normal University. Consent to participate was obtained from each participant.

\section{Consent for publication}

Not applicable. 


\section{Competing interests}

The authors declare that they have no competing interests.

\section{References}

1. Tolar TD, Lederberg AR, Fletcher JM. A structural model of algebra achievement: Computational fluency and spatial visualization as mediators of the effect of working memory on algebra achievement. Educational Psychology. 2009;29(2):239-66.

2. Bryant $P$, Christie $C$, Rendu A. Children's understanding of the relation between addition and subtraction: Inversion, identity, and decomposition. J Exp Child Psychol. 1999;74 3:194-212.

3. Cappelletti M, Kopelman M, Morton J, Butterworth B. Dissociations in Numerical Abilities Revealed by Progressive Cognitive Decline in a Patient with Semantic Dementia. Cognit Neuropsychol. 2005;22:771-93.

4. Dehaene S, Cohen L. Cerebral Pathways for Calculation: Double Dissociation between Rote Verbal and Quantitative Knowledge of Arithmetic. Cortex. 1997;33:2:219-50.

5. Pesenti M, Depoorter N, Seron X. Noncommutability of The N + 0 Arithmetical Rule: A Case Study of Dissociated Impairment. Cortex; a journal devoted to the study of the nervous system and behavior. 2000;36:445-54.

6. Rasmussen $\mathrm{C}, \mathrm{Ho} E$, Bisanz J. Use of the mathematical principle of inversion in young children. J Exp Child Psychol. 2003;85:89-102.

7. Sherman J, Bisanz J. Evidence for Use of Mathematical Inversion By Three-Year-Old Children. Journal of Cognition Development - J COGN DEV. 2007;8:333-44.

8. Delazer M, Domahs F, Lochy A, Karner E, Benke T, Poewe W. Number processing and basal ganglia dysfunction: a single case study. Neuropsychologia. 2004;42 8:1050-62.

9. Ernest $\mathrm{P}$. A model of the cognitive meaning of mathematical expressions. Br J Educ Psychol. 1987;57:343-70.

10. Nakai T, Okanoya K. Neural Evidence of Cross-domain Structural Interaction between Language and Arithmetic. Scientific Reports. 2018;8.

11. Dehaene S, Piazza M, Pinel P, Cohen L. Three Parietal Circuits for Number Processing. Cognit Neuropsychol. 2003;20:487-506.

12. Zhang $\mathrm{H}$, Chen $\mathrm{C}$, Zhou $\mathrm{X}$. Neural correlates of numbers and mathematical terms. Neurolmage. 2012;60:1:230-40.

13. Thioux M, Pesenti M, Costes N, De Volder A, Seron X. Task-independent semantic activation for numbers and animals. Brain research Cognitive brain research. 2005;24:284-90.

14. Arsalidou M, Taylor MJ. Is $2+2=4$ ? Meta-analyses of brain areas needed for numbers and calculations. Neuroimage. 2011;54 3:2382-93. 
15. Cheng DZ, Xiao Q, Chen Q, Cui JX, Zhou XL. Dyslexia and dyscalculia are characterized by common visual perception deficits. Developmental Neuropsychology. 2018;43 6:497-507.

16. Andin J, Fransson P, Dahlstrom O, Ronnberg J, Rudner M. The neural basis of arithmetic and phonology in deaf signing individuals. Lang Cogn Neurosci. 2019;34:7:813-25.

17. Bugden S, Price GR, McLean DA, Ansari D. The role of the left intraparietal sulcus in the relationship between symbolic number processing and children's arithmetic competence. Dev Cogn Neurosci. 2012;2 4:448-57.

18. Bugden S, Woldorff MG, Brannon EM. Shared and distinct neural circuitry for nonsymbolic and symbolic double-digit addition. Hum Brain Mapp. 2019;40 4:1328-43.

19. Kuhl U, Friederici AD, Friederici AD, Emmrich F, Brauer J, Wilcke A, et al. Early cortical surface plasticity relates to basic mathematical learning. Neurolmage. 2020;204:116235.

20. Ashkenazi S, Henik A, Ifergane G, Shelef I. Basic numerical processing in left intraparietal sulcus (IPS) acalculia. Cortex. 2008;44:4:439-48.

21. Baldo JV, Dronkers NF. Neural correlates of arithmetic and language comprehension: A common substrate? Neuropsychologia. 2007;45(2):229-35.

22. Sigmundsson H, Anholt SK, Talcott JB. Are poor mathematics skills associated with visual deficits in temporal processing? Neurosci Lett. 2010;469(2):248-50.

23. Zhou XL, Li MY, Li LNA, Zhang YY, Cui JX, Liu J, et al. The semantic system is involved in mathematical problem solving. Neuroimage. 2018;166:360-70.

24. Frst AJ, Hitch GJ. Separate roles for executive and phonological components of working memory in mental arithmetic. Memory Cognition. 2000;28 5:774-82.

25. Pollack C, Ashby NC. Where arithmetic and phonology meet: The meta-analytic convergence of arithmetic and phonological processing in the brain. Dev Cogn Neurosci. 2018;30:251-64.

26. Li MY, Tan YX, Cui JX, Chen CS, Dong Q, Zhou XL. The Semantic Network Supports Approximate Computation. Neuropsychology. 2019;33:6:842-54.

27. Wu TH, Chen CL, Huang YH, Liu RS, Lee HJ. Effects of long-term practice and task complexity on brain activities when performing abacus-based mental calculations: a PET study. European Journal of Nuclear Medicine Molecular Imaging. 2009.

28. Zhou X, Chen C, Zang Y, Dong Q, Chen C, Qiao S, et al. Dissociated brain organization for single-digit addition and multiplication. Neuroimage. 2007;35(2):871-80.

29. Cheng D, Yan X, Gao Z, Xu K, Zhou X, Chen Q. Common and Distinctive Patterns of Cognitive Dysfunction in Children With Benign Epilepsy Syndromes. Pediatr Neurol. 2017;72:36-41 e1.

30. Tohgi H, Saitoh K, Takahashi S, Takahashi H, Utsugisawa K, Yonezawa H, et al. Agraphia and acalculia after a left prefrontal (F1, F2) infarction. J Neurol Neurosurg Psychiatry. 1995;58:629-32.

31. McCloskey M, Caramazza A, Basili A. Cognitive mechanisms in number processing and calculation: Evidence from dyscalculia. Brain cognition. 1985;4:171-96. 
32. Amalric M, Dehaene S. Origins of the brain networks for advanced mathematics in expert mathematicians. Proc Natl Acad Sci USA. 2016;113 18:4909-17.

33. Amalric M, Dehaene S. A distinct cortical network for mathematical knowledge in the human brain. Neuroimage. 2019;189:19-31.

34. Monti MM, Parsons LM, Osherson DN. Thought Beyond Language: Neural Dissociation of Algebra and Natural Language. Psychol Sci. 2012;23:8:914 - 22.

35. Cappelletti M, Butterworth B, Kopelman M. Spared numerical abilities in a case of semantic dementia. Neuropsychologia. 2001;39:1224-39.

36. Cheng D, Zhou A, Yu X, Chen C, Jia J, Zhou X. Quantifier processing can be dissociated from numerical processing: Evidence from semantic dementia patients. Neuropsychologia. 2013;51 11:2172-83.

37. Anderson JR, Qin YL, Sohn MH, Stenger VA, Carter CS. An information-processing model of the BOLD response in symbol manipulation tasks. Psychon Bull Rev. 2003;10(2):241-61.

38. Geary DC, Hoard MK, Nugent L, Rouder JN. Individual differences in algebraic cognition: Relation to the approximate number and semantic memory systems. J Exp Child Psychol. 2015;140:211-27.

39. Liu J, Yuan L, Chen CS, Cui JX, Zhang H, Zhou XL. The Semantic System Supports the Processing of Mathematical Principles. Neuroscience. 2019;404:102-18.

40. Binder JR, Desai RH, Graves WW, Conant LL. Where Is the Semantic System? A Critical Review and Meta-Analysis of 120 Functional Neuroimaging Studies. Cereb Cortex. 2009;19 12:2767-96.

41. Zhou XL, Li MY, Zhou HT, Li LN, Cui JX. Item-Wise Interindividual Brain-Behavior Correlation in Task Neuroimaging Analysis. Front Neurosci. 2018;12:17.

42. Bradley JV. Complete counterbalancing of immediate sequential effects in a latin square design. J Am Stat Assoc. 1958;53 282:525-8.

43. Dehaene S, Spelke E, Pinel P, Stanescu R, Tsivkin S. Sources of Mathematical Thinking: Behavioral and Brain-Imaging Evidence. Science. 1999;284 5416:970-4.

44. Saxe AM, McClelland JL, Ganguli S. A mathematical theory of semantic development in deep neural networks. Proc Natl Acad Sci USA. 2019;116 23:11537-46.

45. Liu J, Zhang H, Chen $\mathrm{C}$, Chen H, Cui J, Zhou X. The neural circuits for arithmetic principles. Neuroimage. 2017;147:432-46.

46. Huth AG, de Heer WA, Griffiths TL, Theunissen FE, Gallant JL. Natural speech reveals the semantic maps that tile human cerebral cortex. Nature. 2016;532 7600:453-+.

47. Caramazza A, Shelton JR. Domain-specific knowledge systems in the brain: The animate-inanimate distinction. J Cogn Neurosci. 1998;10 1:1-34.

48. Binder JR, Westbury CF, McKiernan KA, Possing ET, Medler DA. Distinct brain systems for processing concrete and abstract concepts. J Cogn Neurosci. 2005;17:6:905 - 17.

49. Hoffman P, Binney RJ, Ralph MAL. Differing contributions of inferior prefrontal and anterior temporal cortex to concrete and abstract conceptual knowledge. Cortex. 2015;63:250-66. 
50. Skipper-Kallal LM, Mirman D, Olson IR. Converging evidence from fMRI and aphasia that the left temporoparietal cortex has an essential role in representing abstract semantic knowledge. Cortex. 2015;69:104-20.

51. Wang J, Conder JA, Blitzer DN, Shinkareva SV. Neural Representation of Abstract and Concrete Concepts: A Meta-Analysis of Neuroimaging Studies. Hum Brain Mapp. 2010;31 10:1459-68.

52. Stanescu-Cosson R, Pinel P, van de Moortele PF, Le Bihan D, Cohen L, Dehaene S. Understanding dissociations in dyscalculia - A brain imaging study of the impact of number size on the cerebral networks for exact and approximate calculation. Brain. 2000;123:2240-55.

53. Dehaene S, Bossini S, Giraux P. The Mental Representation of Parity and Number Magnitude. J Exp Psychol. 1993;122:371-96.

54. Viarouge A, Hubbard EM, Dehaene S. The organization of spatial reference frames involved in the SNARC effect. Q J Exp Psychol. 2014;67 8:1484-99.

55. Knops A, Thirion B, Hubbard EM, Michel V, Dehaene S. Recruitment of an Area Involved in Eye Movements During Mental Arithmetic. Science. 2009;324 5934:1583-5.

56. McCrink K, Dehaene S, Dehaene-Lambertz G. Moving along the number line: Operational momentum in nonsymbolic arithmetic. Percept Psychophys. 2007;69:8:1324-33.

57. Pinhas M, Fischer M. Mental movements without magnitude? A study of spatial biases in symbolic arithmetic. Cognition. 2008;109:408-15.

58. Beaty RE, Kenett YN, Christensen AP, Rosenberg MD, Benedek M, Chen Q, et al. Robust prediction of individual creative ability from brain functional connectivity. Proc Natl Acad Sci USA. 2018;115 5:1087-92.

59. Li M, Cheng D, Lu Y, Zhou X. Neural association between non-verbal number sense and arithmetic fluency. Hum Brain Mapp. 2020;41 18:5128-40.

60. Sommerauer G, Grass K-H, Grabner RH, Vogel SE. The semantic control network mediates the relationship between symbolic numerical order processing and arithmetic performance in children. Neuropsychologia. 2020;141.

61. Lebreton M, Bavard S, Daunizeau J, Palminteri S. Assessing inter-individual differences with taskrelated functional neuroimaging. Nature Human Behaviour. 2019;3 9:897-905.

62. Meyer GJ, Finn SE, Eyde LD, Kay GG, Moreland KL, Dies RR, et al. Psychological testing and psychological assessment - A review of evidence and issues. Am Psychol. 2001;56(2):128-65.

\section{Tables}

\section{Table 1 Brain regions with significantly different activations for algebra and arithmetic.}




\begin{tabular}{|c|c|c|c|c|c|}
\hline \multirow[t]{2}{*}{ Brain region } & \multirow[t]{2}{*}{ Volume } & \multirow[t]{2}{*}{$t$ value } & \multicolumn{3}{|c|}{ Coordinates } \\
\hline & & & $x$ & y & z \\
\hline \multicolumn{6}{|l|}{ Algebra > arithmetic } \\
\hline Right angular gyrus & 37 & 4.56 & 51 & -57 & 36 \\
\hline \multicolumn{6}{|l|}{ Arithmetic > algebra } \\
\hline Left supplementary motor area & 1715 & -3.47 & -15 & 6 & 63 \\
\hline \multirow[t]{3}{*}{ Right middle frontal gyrus } & 1715 & -6.55 & 27 & 42 & 21 \\
\hline & 1715 & -6.34 & 27 & 15 & 12 \\
\hline & 1715 & -6.27 & 15 & 6 & 33 \\
\hline \multirow[t]{3}{*}{ Left insula } & 629 & -6.08 & -33 & 21 & 9 \\
\hline & 629 & -4.78 & -24 & 39 & 33 \\
\hline & 629 & -4.65 & -24 & 3 & 18 \\
\hline Left thalamus & 39 & -5.40 & -21 & -12 & 0 \\
\hline Left cerebellum (9) & 26 & -5.14 & -9 & -42 & -33 \\
\hline \multirow[t]{2}{*}{ Left lingual } & 358 & -5.05 & -3 & -72 & 6 \\
\hline & 358 & -3.89 & -9 & -96 & -3 \\
\hline Left hippocampus & 30 & -4.92 & -36 & -30 & 3 \\
\hline Right postcentral gyrus & 78 & -4.39 & 48 & -33 & 57 \\
\hline \multirow[t]{2}{*}{ Left postcentral gyrus } & 68 & -4.35 & -33 & -42 & 72 \\
\hline & 68 & -4.35 & -21 & -42 & 51 \\
\hline Right postcentral gyrus & 21 & -3.96 & 27 & -36 & 66 \\
\hline Left inferior parietal lobule & 24 & -3.80 & -42 & -42 & 54 \\
\hline
\end{tabular}

Coordinates are in Montreal Neurological Institute space. Extent threshold: $p<.001$, uncorrected, cluster size $>20$.

Table 2 Brain regions with positive and negative brain-behavior correlations for algebra and arithmetic based on single-trial correlations. 
Brain region

Volume $t$ value Coordinates

$x \quad y \quad z$

\section{Positive correlations for algebra}

\begin{tabular}{|c|c|c|c|c|c|}
\hline Left superior frontal gyrus & 56 & 6.38 & -21 & 45 & 39 \\
\hline Right inferior frontal gyrus (Triangle) & 34 & 6.28 & 45 & 33 & 0 \\
\hline Left middle temporal gyrus & 51 & 6.14 & -48 & -21 & -12 \\
\hline \multirow[t]{2}{*}{ Left postcentral gyrus } & 67 & 6.02 & -66 & -21 & 24 \\
\hline & 67 & 5.34 & -63 & -9 & 3 \\
\hline Left middle temporal gyrus & 21 & 5.81 & -66 & -45 & 12 \\
\hline Right middle temporal gyrus & 47 & 5.73 & 60 & -18 & -9 \\
\hline Left superior medial frontal gyrus & 37 & 5.68 & 0 & 63 & 12 \\
\hline \multirow[t]{3}{*}{ Left inferior frontal gyrus (Triangle) } & 201 & 5.65 & -54 & 24 & 3 \\
\hline & 201 & 5.57 & 3 & 33 & -18 \\
\hline & 201 & 5.47 & -54 & 9 & -12 \\
\hline Right superior medial frontal gyrus & 93 & 5.60 & 6 & 45 & 42 \\
\hline Left superior medial frontal gyrus & 93 & 5.11 & -3 & 48 & 15 \\
\hline Left supramarginal gyrus & 31 & 5.20 & -63 & -48 & 30 \\
\hline \multirow[t]{2}{*}{ Left superior frontal gyrus } & 21 & 5.17 & -21 & 63 & 15 \\
\hline & 21 & 5.01 & -33 & 0 & -24 \\
\hline Left supplementary motor area & 20 & 4.63 & -3 & 21 & 60 \\
\hline \multicolumn{6}{|l|}{ Negative correlations for algebra } \\
\hline Right superior parietal lobule & 95 & -8.33 & 24 & -63 & 42 \\
\hline Left superior parietal lobule & 47 & -7.25 & -24 & -63 & 45 \\
\hline Left superior occipital gyrus & 23 & -6.59 & -18 & -90 & 6 \\
\hline Left fusiform gyrus & 20 & -5.15 & -30 & -60 & -12 \\
\hline \multicolumn{6}{|l|}{ Positive correlations for arithmetic } \\
\hline Right inferior temporal gyrus & 20 & 5.82 & 48 & 12 & -39 \\
\hline \multicolumn{6}{|l|}{ Negative correlations for arithmetic } \\
\hline Left superior parietal lobule & 575 & -12.74 & -27 & -63 & 51 \\
\hline
\end{tabular}




\begin{tabular}{|c|c|c|c|c|c|}
\hline & 575 & -7.68 & -39 & -42 & 42 \\
\hline \multirow[t]{2}{*}{ Left precentral gyrus } & 191 & -9.79 & -51 & 0 & 27 \\
\hline & 191 & -4.22 & -60 & 18 & 27 \\
\hline \multirow[t]{3}{*}{ Right middle occipital gyrus } & 666 & -7.91 & 27 & -87 & 15 \\
\hline & 666 & -7.71 & 27 & -66 & 42 \\
\hline & 666 & -6.37 & 45 & -42 & 42 \\
\hline \multirow[t]{3}{*}{ Left middle occipital gyrus } & 735 & -7.75 & -30 & -84 & 12 \\
\hline & 735 & -7.65 & -36 & -69 & -9 \\
\hline & 735 & -6.97 & -12 & -81 & -6 \\
\hline Right middle frontal gyrus & 138 & -7.34 & 36 & 3 & 63 \\
\hline \multirow[t]{2}{*}{ Right precentral gyrus } & 182 & -7.08 & 57 & 12 & 33 \\
\hline & 182 & -6.13 & 54 & 6 & 12 \\
\hline Left middle cingulum gyrus & 34 & -6.83 & -9 & 21 & 39 \\
\hline Left precentral gyrus & 124 & -6.80 & -36 & 0 & 57 \\
\hline Right cerebellum (7) & 73 & -6.57 & 30 & -72 & -51 \\
\hline \multirow[t]{2}{*}{ Left cerebellum (8) } & 140 & -6.34 & -9 & -69 & -30 \\
\hline & 140 & -5.86 & -18 & -72 & -48 \\
\hline Left supplementary motor area & 52 & -5.96 & -6 & 9 & 57 \\
\hline \multirow[t]{2}{*}{ Right fusiform } & 132 & -5.84 & 27 & -78 & -15 \\
\hline & 132 & -5.41 & 42 & -66 & -9 \\
\hline Right inferior temporal gyrus & 61 & -5.71 & 51 & -48 & -9 \\
\hline Left insula & 26 & -5.35 & -30 & 27 & 9 \\
\hline Right inferior frontal gyrus (Triangle) & 40 & -5.27 & 33 & 27 & 27 \\
\hline \multirow[t]{2}{*}{ Right inferior frontal gyrus (Triangle) } & 42 & -5.09 & 51 & 39 & 15 \\
\hline & 42 & -4.86 & 39 & 51 & 27 \\
\hline Left cerebellum (6) & 30 & -5.08 & -30 & -57 & -33 \\
\hline Right calcarine & 20 & -5.07 & 15 & -96 & 3 \\
\hline
\end{tabular}

Coordinates are in Montreal Neurological Institute space. Extent threshold: $p<.001$, uncorrected, cluster size $>20$. 


\section{Figures}

Time

Frame
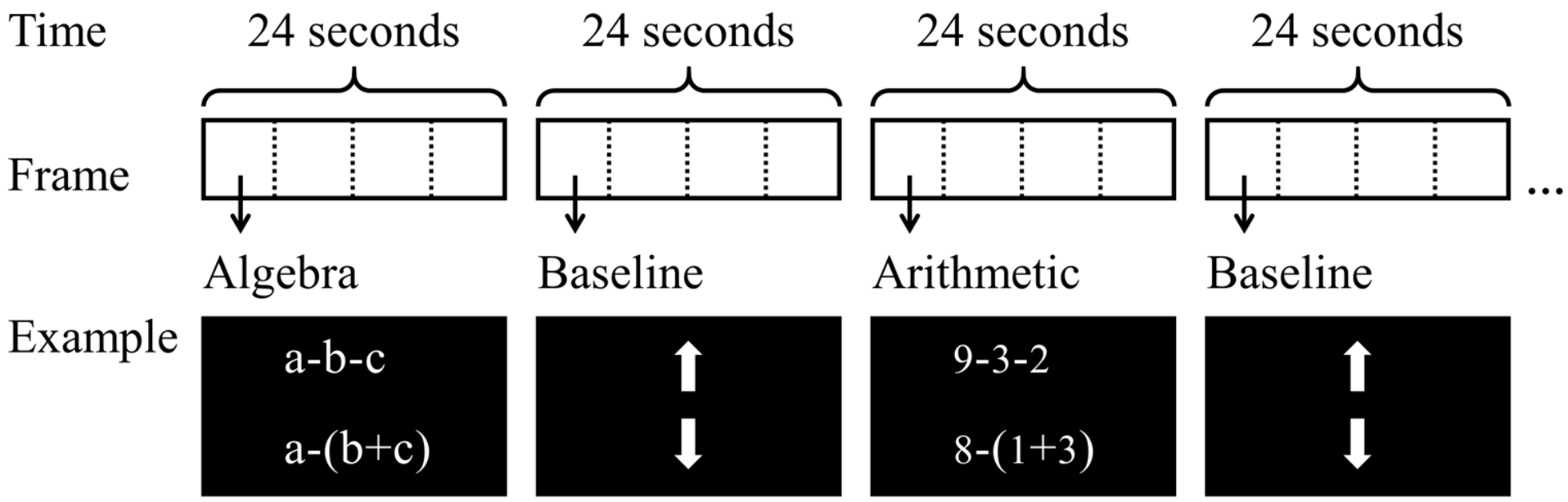

Arithmetic

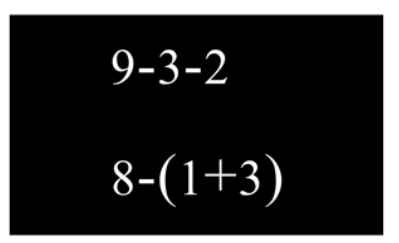

Baseline

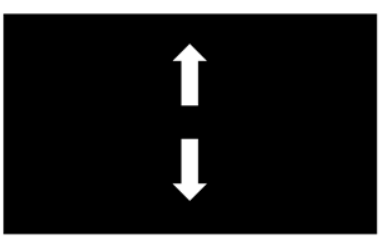

Figure 1

Experimental procedure and examples of trials.

\section{Algebra - baseline}
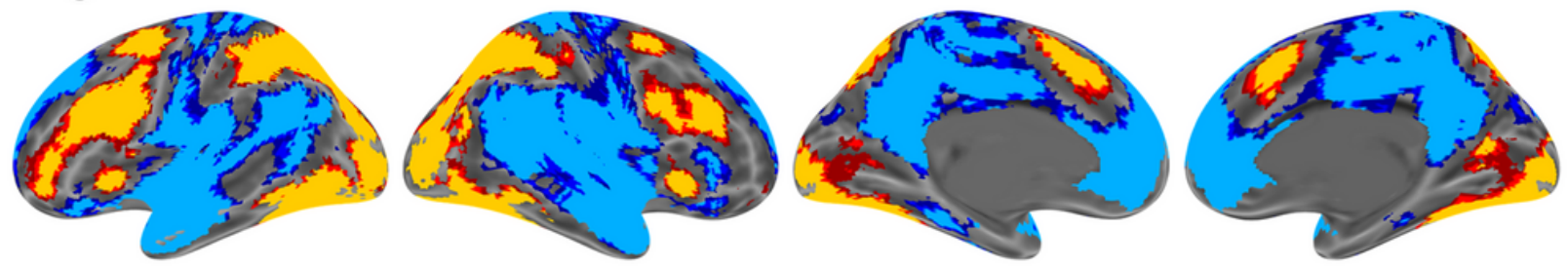

Arithmetic - baseline
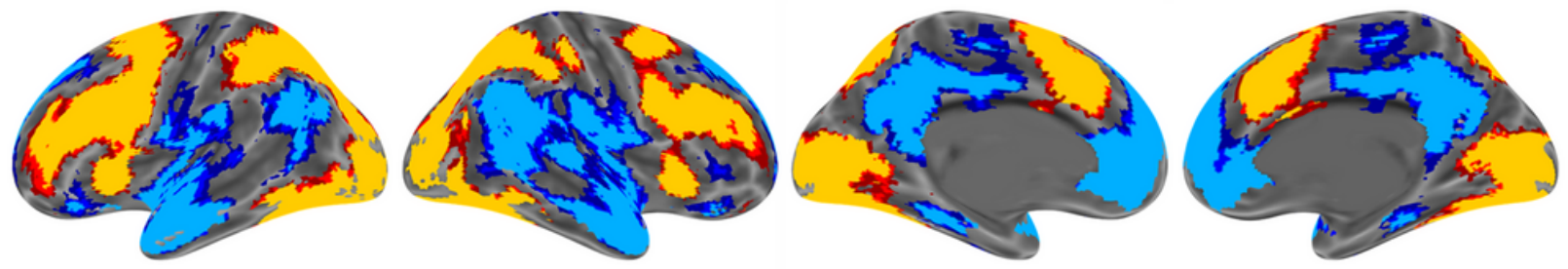

Positive

Algebra - arithmetic
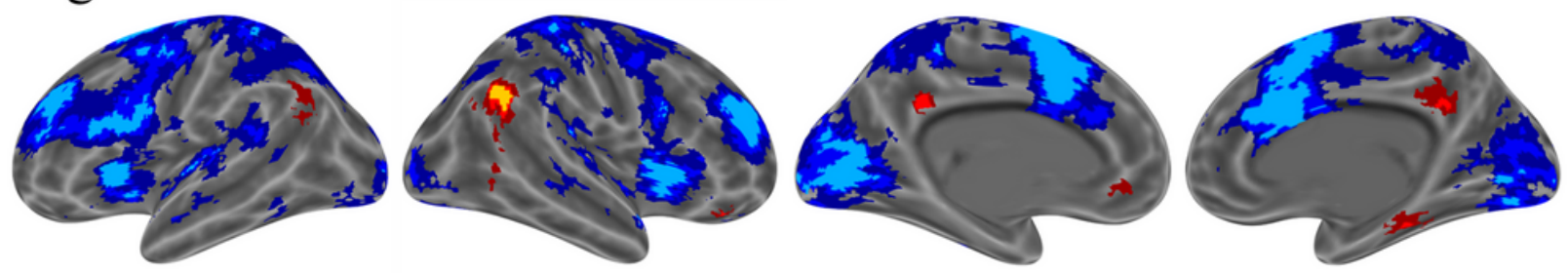

Figure 2

Brain activations for algebra and arithmetic relative to baseline and the contrast between algebra and arithmetic (cluster size $>20$ ). 
Algebra
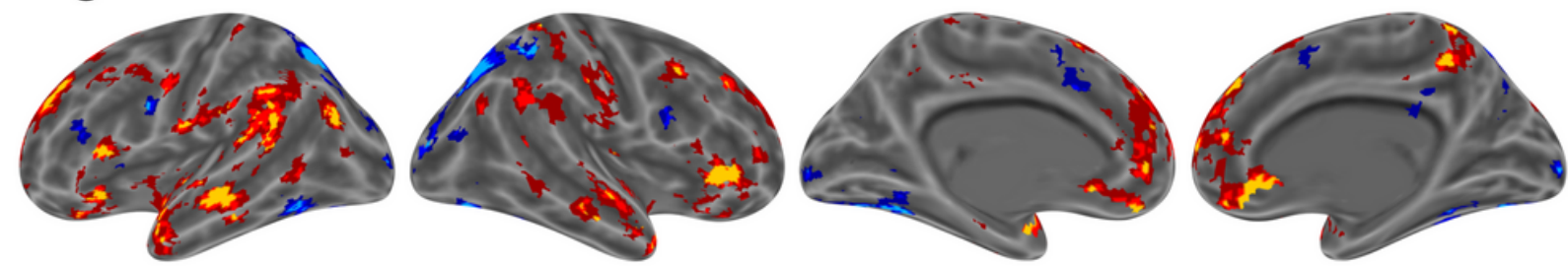

Positive

Arithmetic
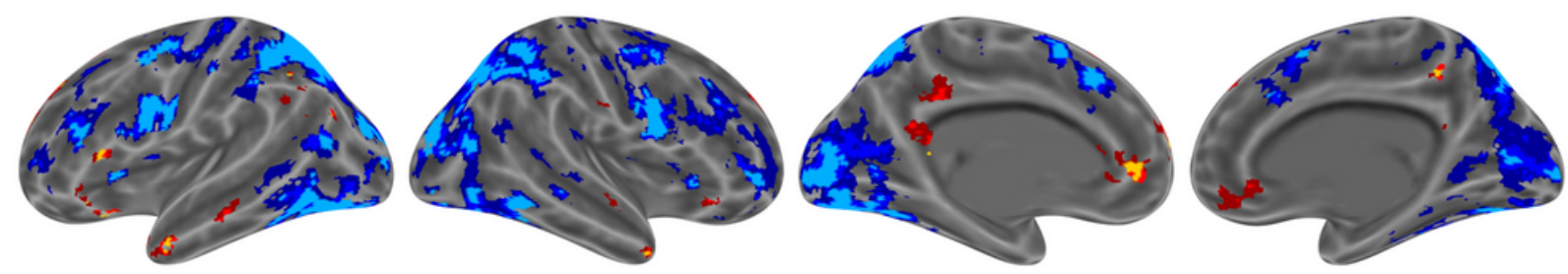

$\square \begin{aligned} & p<.05 \\ & p<.005 \\ & p<.001\end{aligned}$

Negative

$\square \begin{aligned} & p<.05 \\ & p<.005 \\ & p<.001\end{aligned}$

ROIs

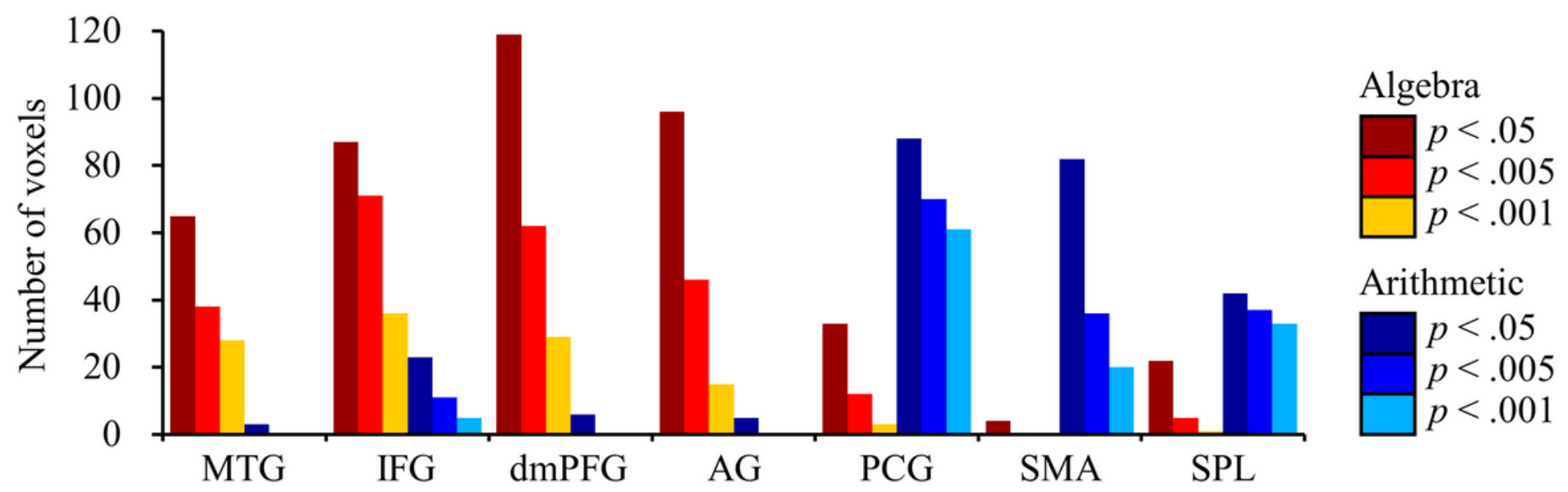

Figure 3

The single-trial brain-behavior correlations for algebra and arithmetic (cluster size $>20$ ) and the number of voxels of the single-trial brain-behavior correlations for algebra and arithmetic in seven ROIs of the semantic, phonological, and visuospatial networks are shown. Semantic network: MTG = middle temporal gyrus; IFG = inferior frontal gyrus; $\mathrm{dmPFC}=$ dorsomedial prefrontal cortex; $\mathrm{AG}=$ angular gyrus. Phonologic network: $P C G$ = precentral gyrus; $S M A$ = supplementary motor area; visuospatial network: $\mathrm{SPL}=$ superior parietal lobule. 


\section{Algebra}
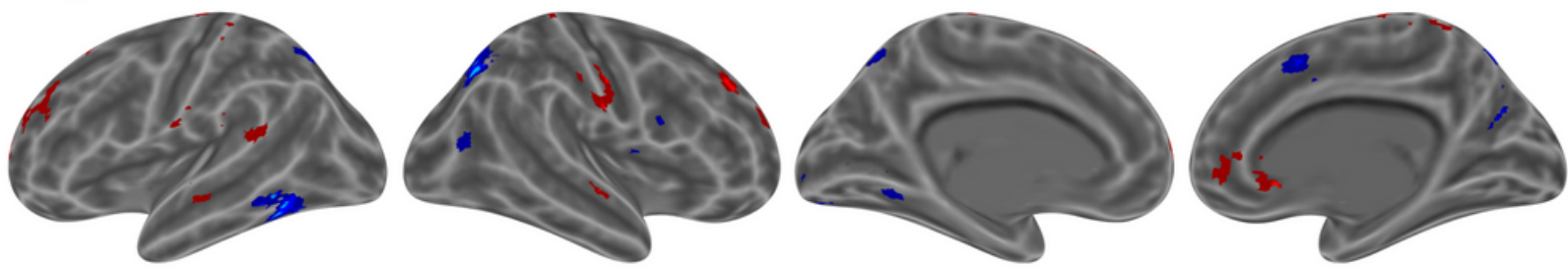

Positive

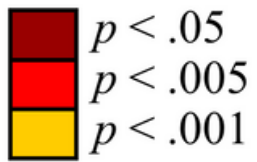

\section{Arithmetic}
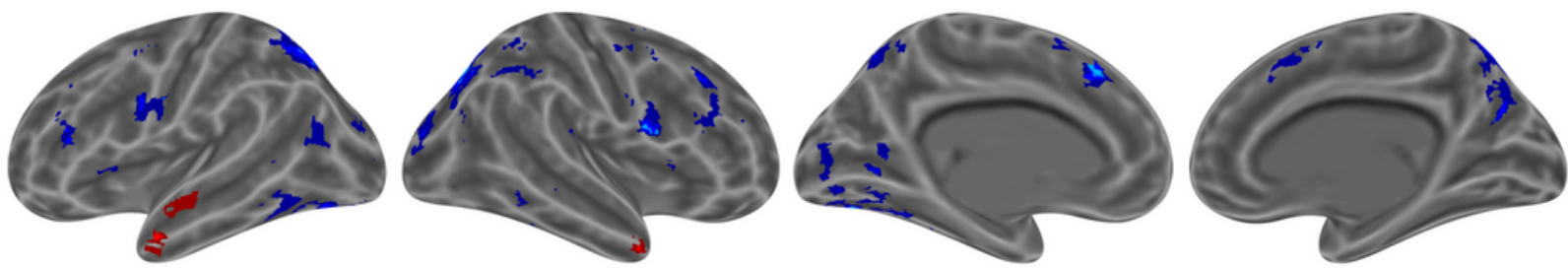

Negative

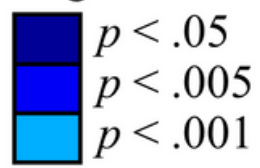

\section{ROIs}

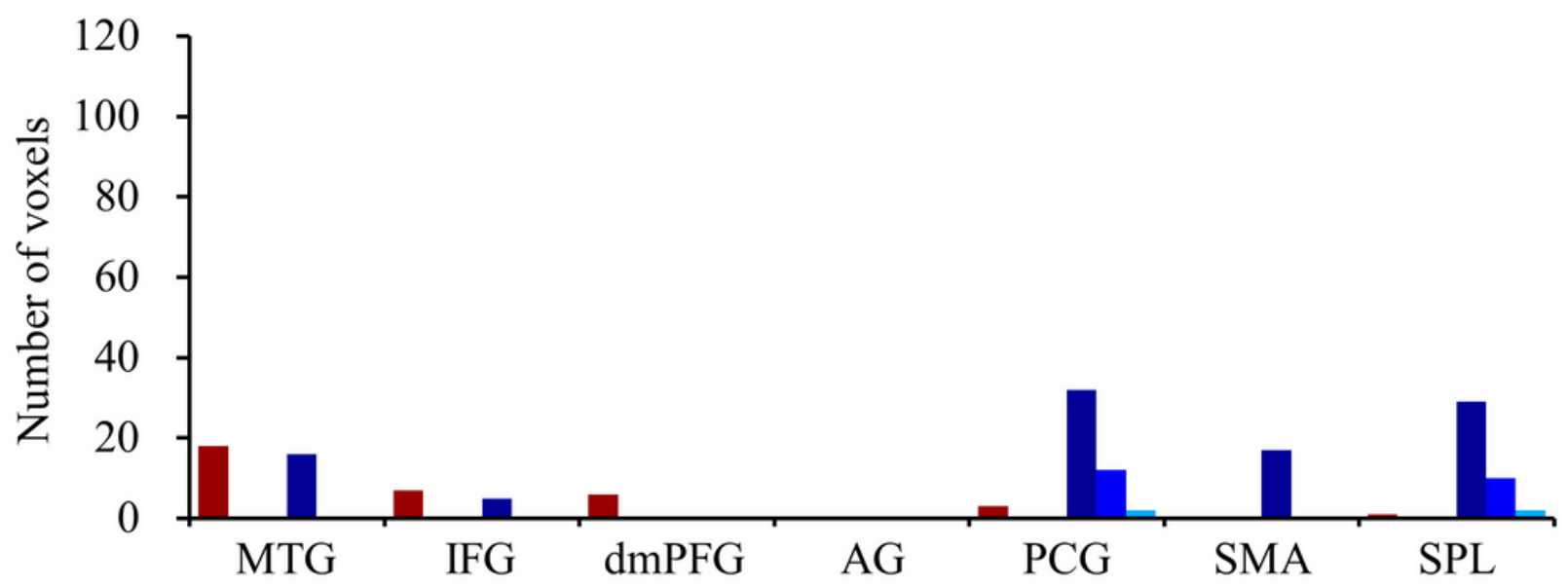

Algebra

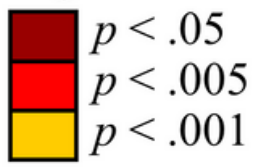

Arithmetic

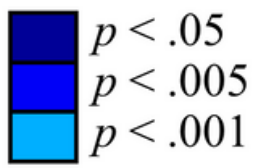

\section{Figure 4}

The mean-trial brain-behavior correlations for algebra and arithmetic (cluster size $>20$ ) and the number of voxels of the mean-trial brain-behavior correlations for algebra and arithmetic in seven ROls of the semantic, phonological, and visuospatial networks are shown. Semantic network: MTG = middle temporal gyrus; IFG = inferior frontal gyrus; $d m P F C=$ dorsomedial prefrontal cortex; $A G$ = angular gyrus . Phonologic network: $P C G$ = precentral gyrus; $S M A$ = supplementary motor area. Visuospatial network: $\mathrm{SPL}=$ superior parietal lobule. 